\title{
Ethiopian muscids (Diptera, Muscidae) egg-carriers of Stylogaster Macquart (Diptera, Conopidae)
}

\author{
MÁRCIA S. COURI ${ }^{1}$, KURT JORDAENS ${ }^{2}$, LORE GEERAERT ${ }^{3,4}$, RAFAEL $^{3,5}$ \\ MATHEUS $^{1,5}$ and ANGELINA P. VIEIRA-ARAÚJO ${ }^{1,6}$ \\ ${ }^{1}$ Museu Nacional, Quinta da Boa Vista, São Cristóvão, 20940-040 Rio de Janeiro, RJ, Brazil \\ ${ }^{2}$ Royal Museum for Central Africa, Department of Biology, Leuvensesteenweg 13, B-3080 Tervuren, Belgium \\ ${ }^{3}$ Plant Conservation and Population Biology, University of Leuven, Kasteelpark Arenberg 31-2435, BE-3001 Leuven, Belgium \\ ${ }^{4}$ Biodiversity Inventory for Conservation (BINCO), Walmersumstraat 44, BE-3380 Glabbeek, Belgium \\ ${ }^{5}$ PIBIC/CNPq, SHIS QI 1, Conjunto B, Blocos A, B, C e D, Lago Sul, 71605-001 Brasília, DF, Brazil \\ ${ }^{6}$ PIBIC/UFRJ, Prédio da Reitoria, $8^{\circ}$ andar, Sala 811, Ilha da Cidade Universitária, 21941-590 Rio de Janeiro, RJ, Brazil
}

Manuscript received on August 31, 2018; accepted for publication on November 30, 2018

\begin{abstract}
How to cite: COURI MS, JORDAENS K, GEERAERT L, MATHEUS R AND VIEIRA-ARAÚJO AP. 2019. Ethiopian muscids (Diptera, Muscidae) egg-carriers of Stylogaster Macquart (Diptera, Conopidae). An Acad Bras Cienc 91: e20180901. DOI 10.1590/0001-3765201920180901.
\end{abstract}

\begin{abstract}
We here report for the first time on the presence of three species of the conopid genus Stylogaster Macquart (Diptera, Conopidae) in Ethiopia, viz. S. nitens Brunetti, S. westwoodi Smith and Stylogaster sp.. We further screened 908 muscid flies (Diptera, Muscidae) for the presence of impaled eggs of Stylogaster and recorded eggs on 89 individuals (9.8\%). Eggs were impaled on eight species, viz. Limnophora translucida Stein, Musca lusoria Wiedemann, Musca splendens Pont, Neomyia chrysopyga (Emden), Pseudohelina nigritarsis (Jaennicke), Stomoxys omega Newstead, Stomoxys taeniatus Bigot and Stomoxys varipes (Bezzi). The maximum number of eggs found on a single muscid was six. We illustrated the dissected eggs. L. translucida, M. lusoria, M. splendens, N. chrysopyga and S. varipes are reported as new muscid hosts species for Stylogaster.
\end{abstract}

Key words: Afrotropical region, dart-eggs, Ethiopia, new record.

\section{INTRODUCTION}

Conopidae (Insecta, Diptera), also known as thickheaded-flies, and their larvae are internal parasites mainly in Hymenoptera. Females of the genus Stylogaster Macquart (subfamily Stylogastrinae) have a unique oviposition behaviour during which they bend the long and thin abdomen forward to 'throw' their eggs as darts against various body parts of other insects (hence their vernacular name,

Correspondence to: Márcia Souto Couri

E-mail: courimarcia@gmail.com

ORCid: https://orcid.org/0000-0002-5340-395X dart-egg-flies), especially of other brachycerous Diptera, and Orthoptera (cockroaches and crickets). The biology of the genus was discussed by, e.g., Stuckenberg (1963) and Smith (1967) while Couri and Barros (2010) summarized available data on calyptrate Diptera Stylogaster hosts. Stylogaster larvae are endoparasites in Orthoptera, especially cockroaches and crickets (Skevington et al. 2010). Even though impaled eggs of Stylogaster so far have been recorded from 30 species of Muscidae, Stuke (2012) suggests that Calyptratae are not normal larval hosts since no larva are found internally. 
Muscid hosts are probably egg-carriers since no conopid has been reared from a muscid species (see Stuke 2017: XXV for a discussion). In Africa, the egg-darting behavior is usually associated with the presence of army ants as, when they pass, other insects fly away and become vulnerable to the darted eggs of Stylogaster females which hover over the army ants. It is also believed that Stylogaster females are attracted to dung, darting their eggs on other Diptera attracted by the faeces (Stuckenberg 1963, Couri and Pont 2006, Couri and Barros 2010).

Smith (1980) listed 14 Stylogaster species for the Afrotropical Region, but recently Stuke (2012) added 21 new species to the genus and also presented new faunistic records for nine previously described species, an identification key to the Afrotropical species and a checklist with a revised distribution. Of the 35 Afrotropical Stylogaster species, 22 are endemic to Madagascar. More recently, Stuke (2017) provided an updated catalogue of the genus Stylogaster and reported a total of 125 species worldwide of which 43 are found in the Afrotropical region. Only in the Neotropical region more (73) species have been recorded.

\section{MATERIALS AND METHODS}

Muscidae were sampled in February and March 2016 with insect nets and malaise traps (black, British museum type) in two locations in the highlands of SW Ethiopia, viz. the Limmu Coffee Farm (Horizon Plantations P.L.C.) and the Girmo Forest complex. Both are in the Limmu-Kosa area of the Jimma zone, ca. $50 \mathrm{~km} \mathrm{~N}$ of Jimma. The Limmu Coffee Farm is a shaded coffee (Coffea arabica Linnaeus, 1753) plantation with a cleared understory and a low density of large canopy trees, dominantly Acacia spp. and Albizia schimperiana Oliver, 1871. The Girmo Forest complex is a semi-natural montane forest where C. arabica grows naturally. The forest has a dense and species rich understory and canopy layer, with
Olinia rochetiana Jussieu, 1846, Aningeria adolffriederici (Engler) Robyns and Gilbert, 1947, Dracaena steudneri Engler, 1895 and Ficus spp. as the dominant tree species. The collected muscides were stored in $70 \% \mathrm{v} / \mathrm{v}$ ethanol and exported to Belgium (export permit: EBI71/5065/2016). Upon arrival, they were pinned, labelled. and stored at the Plant Conservation and Population Biology unit of the University of Leuven.

Muscidae were identified by MC using keys in Emden (1951), Zielke (1971) and Zumpt (1973). The flies were screened for the presence of Stylogaster eggs and if present, eggs were counted and the body part(s) to which they were attached was (were) noted. In order to allow dissection of the Stylogaster eggs, several pinned muscid specimens were kept in a moist chamber for 24 hours. Afterwards, eggs were removed using entomological pins and macerated in a solution of $10 \%$ potassium hydroxide for 24 hours. After identification and making color photos using a Syncroscopy Auto-Montage with a Leica MZ16 optical microscope, eggs were stored in a microtube with glycerol attached to the pin of the pinned specimens (for more information on the procedure, see Couri and Barros, 2010). Eggs and adult Stylogaster were identified using Smith (1967) and Stucke (2012).

\section{RESULTS AND DISCUSSION}

A total number of 908 Muscidae specimens were identified and checked for the presence of Stylogaster eggs. Stylogaster eggs were found on 89 specimens $(9.8 \%)$ of eight muscid species, viz. Limnophora translucida Stein, 1913; Musca lusoria Wiedemann, 1824; Musca splendens Pont, 1980; Neomyia chrysopyga (Emden, 1939); Pseudohelina nigritarsis (Jaennicke, 1867); Stomoxys omega Newstead, 1907; Stomoxys taeniatus Bigot, 1888 and Stomoxys varipes (Bezzi, 1907). The three Stomoxys species accounted for $90 \%$ of the impaled specimens $(85.4 \%)$ with $S$. omega $(85.4 \%)$ and $S$. taeniatus (9\%) showing the 
highest frequences. Frequencies of other impaled muscids were very low $(<3.5 \%)$.

Most of the impaled muscids were females (76/89 or $85.4 \%$ )(Table I). A similar high prevalence for females was observed by Couri and Barros (2010) and Couri et al. (2013). Several Stylogaster eggs were found on the ventral part of the abdomen of the muscid hosts and this suggests that eggs were not darted during the flight of the hosts fleeing the army ants. More likely, Stylogaster females visit dung where they dart their eggs on hosts that are attracted to dung, such as Stomoxys spp. This might also explain the high number of Stomoxys female Muscidae infested with eggs compared to males. The maximum number of eggs found on a single muscid specimen was highly variable and ranged from one to six (a female of $S$. omega) (Table II).

We identified three Stylogaster species, viz. S. nitens Brunetti, S. westwoodi Smith and Stylogaster sp. Both S. nitens and S. westwoodi are widely distributed in the Afrotropical Region, and previously have been recorded from Angola, Democratic Republic of Congo, Ghana, South Africa, Uganda and from Democratic Republic of Congo, Kenya, Malawi, Nigeria, South Africa, Tanzania, Zambia and Zimbabwe, respectively.

Illustrations of the dissected eggs are depicted in Figs 1f and 1g. One of the eggs is probably of $S$. nitens (Fig. 1f) because adults of this species were collected along with the muscid specimens impaled with Stylogaster eggs. The other eggs showed high morphological resemblance with the egg of an unknown Stylogaster sp. figured by Smith (1967, Figs 16 and 17 therein).

In conclusion, we here record, for the first time, the presence of three species of the conopid genus Stylogaster, in Ethiopia, and record the muscid species Limnophora translucida, Musca lusoria, Musca splendens, Neomyia chrysopyga and Stomoxys varipes as new hosts for Stylogaster species.

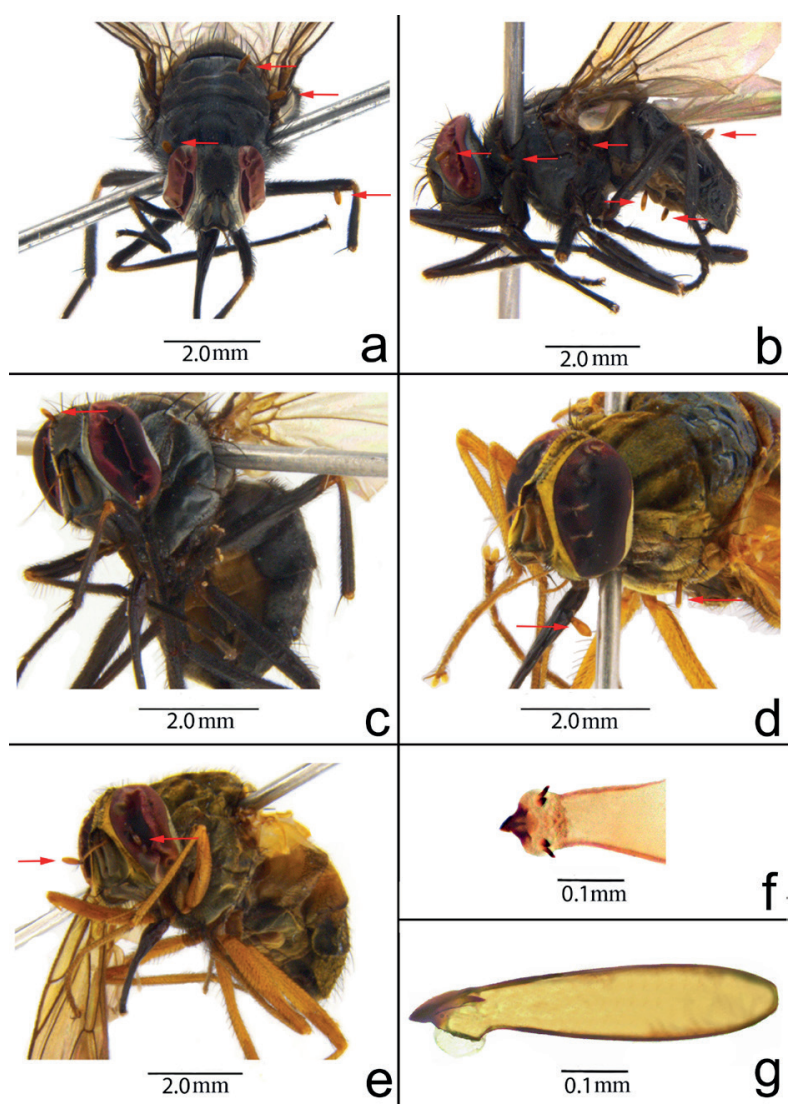

Figure 1 - a) Lateral view of Stomoxys omega Newstead (ENT000027971, adult §); b) Lateral view of Stomoxys omega Newstead (ENT000028668, adult + ); c) Lateral view of Stomoxys omega Newstead (ENT000027797, adult $\bigcirc$ ); d) Lateral view of Stomoxys taeniatus Bigot (ENT000028471, adult $\widehat{\jmath})$; e) Lateral view of Stomoxys taeniatus Bigot (ENT000028756, adult §); f) Ventral view of the anterior part of the egg of Stylogaster nitens Brunetti; Figure g) Lateral view of the egg of Stylogaster sp.

\section{MATERIAL EXAMINED}

\section{Muscidae}

Limnophora translucida Stein - ETHIOPIA: Jimma, Girmo, 0752'49”N, 3651'51'E, 19.ii.2016, 1961 m, coll. L. Geeraert, Random sweepnetting, Semi-forest coffee management system, 10 , ENT000028289.

Musca lusoria Wiedemann - ETHIOPIA: Jimma, Limmu-Kosa, 0757'12'N, 3652'46”E, 9.ii.2016, 1777 m; coll. L. Geeraert, Random sweepnetting, Plantation coffee management system, 1\%, ENT000027990; 19, ENT000027954; 
TABLE I

Muscidae species as Stylogaster (Conopidae) hosts in Ethiopia.

\begin{tabular}{lccc}
\hline \multicolumn{1}{c}{ Muscidae species } & $\mathbf{N}^{\circ}$ of males & $\mathbf{N}^{\circ}$ of females & Range of no of eggs on a single host \\
\hline Limnophora translucida & 1 & 0 & 1 \\
Musca lusoria & 0 & 3 & 1 \\
Musca splendens & 0 & 1 & 1 \\
Neomyia chrysopyga & 0 & 1 & 1 \\
Pseudohelina nigritarsis & 3 & 0 & 1 \\
Stomoxys omega & 7 & 64 & $1-6$ \\
Stomoxys taeniatus & 2 & 6 & $1-2$ \\
Stomoxys varipes & 0 & 1 & 1 \\
TOTAL & 13 & 76 & \\
\hline
\end{tabular}

TABLE II

Muscidae host of Stylogaster spp. with details on number of eggs and body part where impaled were recorded.

\begin{tabular}{|c|c|c|c|c|c|}
\hline Muscidae species & Identification $\mathrm{nr}$ & Locality & Sex & Part of body impaled & $\begin{array}{l}\text { Nr of } \\
\text { eggs }\end{array}$ \\
\hline $\begin{array}{l}\text { Limnophora translucida } \\
\text { Stein }\end{array}$ & ENT000028289 & Girmo & $\hat{\sigma}$ & Tergite 5 dorsal & 1 \\
\hline Musca lusoria Wiedemann & ENT000027990 & Limmu-Kosa & ㅇ & Back of left eye & 1 \\
\hline Musca lusoria Wiedemann & ENT000027954 & Limmu-Kosa & q & Tergite 4 dorsal & 1 \\
\hline Musca lusoria Wiedemann & ENT000028811 & Limmu-Kosa & q & Right anepistenum & 1 \\
\hline Musca splendens Pont & ENT000028275 & Girmo & q & Left anepimeron & 1 \\
\hline $\begin{array}{l}\text { Neomyia chrysopyga } \\
\text { (Emden) }\end{array}$ & ENT000027926 & Limmu-Kosa & 우 & Left katepisternum & 1 \\
\hline $\begin{array}{l}\text { Pseudohelina nigritarsis } \\
\text { (Jaennicke) }\end{array}$ & ENT000028063 & Girmo & $\hat{\sigma}$ & Tergite 4 left lateral & 1 \\
\hline $\begin{array}{l}\text { Pseudohelina nigritarsis } \\
\text { (Jaennicke) }\end{array}$ & ENT000027757 & Girmo & $\hat{0}$ & Right eye & 1 \\
\hline $\begin{array}{l}\text { Pseudohelina nigritarsis } \\
\text { (Jaennicke) }\end{array}$ & ENT000028393 & Girmo & $\hat{\sigma}$ & Left eye & 1 \\
\hline Stomoxys omega Newstead & ENT000028080 & Girmo & ㅇ & $\begin{array}{l}\text { Right greater ampulla; base of right wing; } \\
\text { right anepimerum; tergite } 5 \text { left lateral; } \\
\text { tergite } 1+2 \text { ventral }\end{array}$ & 5 \\
\hline Stomoxys omega Newstead & ENT000028071 & Girmo & q & Left wing insertion & 1 \\
\hline Stomoxys omega Newstead & ENT000028052 & Girmo & q & Right anepisternum & 1 \\
\hline Stomoxys omega Newstead & ENT000028051 & Girmo & 우 & $\begin{array}{c}\text { Right notopleurum; left katepisterno; } \\
\text { meron; } \\
\text { tergite } 3 \text { ventral }\end{array}$ & 4 \\
\hline Stomoxys omega Newstead & ENT000028050 & Girmo & q & Right notopleurum & 1 \\
\hline Stomoxys omega Newstead & ENT000028006 & Girmo & q & Left eye & 1 \\
\hline Stomoxys omega Newstead & ENT000028022 & Girmo & $\pi$ & Right notopleurum & 1 \\
\hline Stomoxys omega Newstead & ENT000027971 & Girmo & $\hat{0}$ & $\begin{array}{l}\text { Postpronotum 1; escuto dorsal; scutum; } \\
\text { apex of right fêmur }\end{array}$ & 4 \\
\hline Stomoxys omega Newstead & ENT000027970 & Girmo & 우 & $\begin{array}{l}\text { Left postpronotum; bellow the anterior } \\
\text { spiracle; } \\
\text { Left meron }\end{array}$ & 3 \\
\hline
\end{tabular}


TABLE II (continuation)

\begin{tabular}{|c|c|c|c|c|c|}
\hline Muscidae species & Identification nr & Locality & Sex & Part of body impaled & $\begin{array}{l}\text { Nr of } \\
\text { eggs }\end{array}$ \\
\hline Stomoxys omega Newstead & ENT000027968 & Girmo & q & Left eye & 1 \\
\hline Stomoxys omega Newstead & ENT000027966 & Girmo & q & Right notopleurum; left anepisternum & 2 \\
\hline Stomoxys omega Newstead & ENT000027956 & Limmu-Kosa & $\hat{\sigma}$ & Right eye & 1 \\
\hline Stomoxys omega Newstead & ENT000028724 & Girmo & q & Right anterior fêmur & 1 \\
\hline Stomoxys omega Newstead & ENT000028717 & Girmo & $\hat{o}$ & Left notopleurum; left eye; tergite 3 dorsal & 3 \\
\hline Stomoxys omega Newstead & ENT000028685 & Girmo & q & Pre-scutum, lateral left & 1 \\
\hline Stomoxys omega Newstead & ENT000028683 & Girmo & q & Left eye; left notopleurum & 2 \\
\hline Stomoxys omega Newstead & ENT000028682 & Girmo & q & left notopleurum & 1 \\
\hline Stomoxys omega Newstead & ENT000028681 & Girmo & q & Left anepisternum & 1 \\
\hline Stomoxys omega Newstead & ENT000028680 & Girmo & q & Left notopleurum; base of right wing & 2 \\
\hline Stomoxys omega Newstead & ENT000028676 & Girmo & q & Left notopleurum; left katepisternum & 2 \\
\hline Stomoxys omega Newstead & ENT000028675 & Girmo & q & Tergite 4 dorsal & 1 \\
\hline Stomoxys omega Newstead & ENT000028671 & Girmo & q & Left notopleurum; left anepisternum & 2 \\
\hline Stomoxys omega Newstead & ENT000028668 & Girmo & q & $\begin{array}{l}\text { Left eye; anterior left spiracle; posterior } \\
\text { left spiracle; tergite } 3 \text { ventral; two in } \\
\text { tergite } 4 \text {, ventral and dorsal }\end{array}$ & 6 \\
\hline Stomoxys omega Newstead & ENT000028666 & Girmo & q & Right katepisternum & 1 \\
\hline Stomoxys omega Newstead & ENT000028663 & Girmo & q & Left katepisternum & 1 \\
\hline Stomoxys omega Newstead & ENT000028661 & Girmo & q & Right notopleurum & 1 \\
\hline Stomoxys omega Newstead & ENT000028651 & Girmo & q & Right eye & 1 \\
\hline Stomoxys omega Newstead & ENT000028649 & Girmo & q & Right eye & 1 \\
\hline Stomoxys omega Newstead & ENT000028647 & Girmo & q & Left notopleurum; left eye & 2 \\
\hline Stomoxys omega Newstead & ENT000028643 & Girmo & q & Right anepisternum; base of right wing & 2 \\
\hline Stomoxys omega Newstead & ENT000028642 & Girmo & q & Right postpronotum & 1 \\
\hline Stomoxys omega Newstead & ENT000028640 & Girmo & q & Right prescutum & 1 \\
\hline Stomoxys omega Newstead & ENT000028637 & Girmo & q & Tergite $1+2$ dorsal & 1 \\
\hline Stomoxys omega Newstead & ENT000028633 & Girmo & q & Tergite 4 dorsal & 1 \\
\hline Stomoxys omega Newstead & ENT000028630 & Girmo & $\hat{0}$ & Scutum; right eye & 2 \\
\hline Stomoxys omega Newstead & ENT000028625 & Girmo & q & Left eye & 1 \\
\hline Stomoxys omega Newstead & ENT000028620 & Girmo & q & Left postpronotum & 1 \\
\hline Stomoxys omega Newstead & ENT000028616 & Girmo & q & Above postalar wall; base of left wing & 2 \\
\hline Stomoxys omega Newstead & ENT000028615 & Girmo & q & Right katepisternum & 1 \\
\hline Stomoxys omega Newstead & ENT000028606 & Girmo & q & Scutum & 1 \\
\hline Stomoxys omega Newstead & ENT000028589 & Girmo & q & Tergite 2 dorsal & 1 \\
\hline Stomoxys omega Newstead & ENT000028586 & Girmo & q & Left anepimerum & 1 \\
\hline Stomoxys omega Newstead & ENT000028577 & Girmo & q & Prescutum & 1 \\
\hline Stomoxys omega Newstead & ENT000028575 & Girmo & q & Left eye & 1 \\
\hline Stomoxys omega Newstead & ENT000028513 & Girmo & q & Left eye; scutum & 2 \\
\hline Stomoxys omega Newstead & ENT000028503 & Girmo & q & Tergite $1+2$ right lateral & 1 \\
\hline Stomoxys omega Newstead & ENT000028502 & Girmo & q & Tergite 1 ventral & 1 \\
\hline Stomoxys omega Newstead & ENT000028493 & Girmo & q & Right eye & 1 \\
\hline
\end{tabular}


TABLE II (continuation)

\begin{tabular}{|c|c|c|c|c|c|}
\hline Muscidae species & Identification $\mathrm{nr}$ & Locality & Sex & Part of body impaled & $\begin{array}{l}\text { Nr of } \\
\text { eggs }\end{array}$ \\
\hline Stomoxys omega Newstead & ENT000028491 & Girmo & ㅇ & Right postpronotum; left katepisternum & 2 \\
\hline Stomoxys omega Newstead & ENT000028489 & Girmo & 우 & Scutum & 1 \\
\hline Stomoxys omega Newstead & ENT000028488 & Girmo & 우 & Right eye; left anepimerum & 2 \\
\hline Stomoxys omega Newstead & ENT000028485 & Girmo & o & Left anepisternum & 1 \\
\hline Stomoxys omega Newstead & ENT000028482 & Girmo & o & Left spiracle & 1 \\
\hline Stomoxys omega Newstead & ENT000027746 & Girmo & 우 & Tergite $1+2$ ventral; tergite 3 ventral & 2 \\
\hline Stomoxys omega Newstead & ENT000027748 & Girmo & $\hat{0}$ & Base of left wing & 1 \\
\hline Stomoxys omega Newstead & ENT000027763 & Gima & 우 & Right notopleuron & 1 \\
\hline Stomoxys omega Newstead & ENT000027771 & Girmo & ㅇ & Left anterior spiracle; tergite 5 dorsal & 2 \\
\hline Stomoxys omega Newstead & ENT000027773 & Girmo & q & Right eye & 1 \\
\hline Stomoxys omega Newstead & ENT000027792 & Girmo & q & Left eye & 1 \\
\hline Stomoxys omega Newstead & ENT000027795 & Girmo & 우 & Left notopleurum; tergite $1+2$ ventral & 2 \\
\hline Stomoxys omega Newstead & ENT000027797 & Girmo & 우 & Frons & 1 \\
\hline Stomoxys omega Newstead & ENT000027800 & Girmo & 우 & $\begin{array}{l}\text { Left eye; right postpronotum; left upper } \\
\text { calypter }\end{array}$ & 3 \\
\hline Stomoxys omega Newstead & ENT000027837 & Girmo & 우 & $\begin{array}{l}\text { Left postpronotum; tergite } 1+2 \text { right } \\
\text { lateral }\end{array}$ & 2 \\
\hline Stomoxys omega Newstead & ENT000027864 & Girmo & 우 & Anepisternum & 1 \\
\hline Stomoxys omega Newstead & ENT000027868 & Girmo & $\hat{0}$ & Right anepisternum & 1 \\
\hline Stomoxys omega Newstead & ENT000027874 & Girmo & o & Prescutum; scutum & 2 \\
\hline Stomoxys omega Newstead & ENT000027908 & Girmo & 우 & Postpronotum & 1 \\
\hline Stomoxys omega Newstead & ENT000027913 & Girmo & q & Scutum; anepisternum & 2 \\
\hline Stomoxys omega Newstead & ENT000028352 & Girmo & 우 & Tergite 4 dorsal & 1 \\
\hline Stomoxys omega Newstead & ENT000028456 & Girmo & 우 & $\begin{array}{l}\text { Right postpronotum; right notopleurum; } \\
\text { left anepisternum; apex of anterior femus; } \\
\text { tergite } 5 \text { dorsal }\end{array}$ & 5 \\
\hline Stomoxys omega Newstead & ENT000028253 & Girmo & o & Right eye & 1 \\
\hline Stomoxys taeniatus Bigot & ENT000027953 & $\begin{array}{l}\text { Limmu } \\
\text { u-Kosa }\end{array}$ & o & Left eye & 1 \\
\hline Stomoxys taeniatus Bigot & ENT000028756 & Limmu-Kosa & 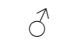 & Right eye; left eye & 2 \\
\hline Stomoxys taeniatus Bigot & ENT000028618 & Girmo & 우 & Left postpronotum & 1 \\
\hline Stomoxys taeniatus Bigot & ENT000028471 & Limmu-Kosa & $\delta$ & Proboscis; right anepisternum & 2 \\
\hline Stomoxys taeniatus Bigot & ENT000027784 & Limmu-Kosa & 우 & Left prescutum & 1 \\
\hline Stomoxys taeniatus Bigot & ENT000027785 & Limmu-Kosa & 우 & Right eye & 1 \\
\hline Stomoxys taeniatus Bigot & ENT000027873 & Girmo & 우 & Right anepisternum & 1 \\
\hline Stomoxys taeniatus Bigot & ENT000028317 & Limmu-Kosa & 우 & Tergite 4 dorsal & 1 \\
\hline Stomoxys varipes (Bezzi) & ENT000028547 & Girmo & 우 & Tergite 5 dorsal & 1 \\
\hline
\end{tabular}

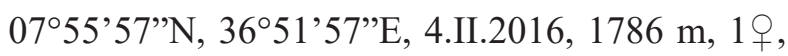
ENT000028811.

Musca splendens Pont - ETHIOPIA: Jimma, Girmo, 0752'49”N, 3651'51'E, 19.ii.2016, 1961 m, coll. L. Geeraert, Random sweepnetting,
Semi-forest coffee management system, 1우, ENT000028275.

Neomyia chrysopyga (Emden) - ETHIOPIA: Jimma, Limmu-Kosa, 0757'12'N, 3652'46”E, 9.ii.2016, 1777 m, coll. L. Geeraert, Random 
sweepnetting, Plantation coffee management system, 1 , ENT000027926.

Pseudohelina nigritarsis (Jaennicke) ETHIOPIA: Jimma, Girmo, 0752’49”N, 3651'51'E, 19.ii.2016, $1961 \mathrm{~m}$, coll. L. Geeraert, Random sweepnetting, Semi-forest coffee management system, 1ð, ENT000028063; 1ð, ENT000027757; 10̊, ENT000028393.

Stomoxys omega Newstead (Figs 1ac) - ETHIOPIA: Jimma, Girmo, 07052'51'N, 3651'47'E, 24.ii.2016, $1920 \mathrm{~m}$, coll. L. Geeraert, Random sweep netting, Semi-forest coffee management system, 2, ENT000027746, ENT000027748; 4 ㅇ ENT000027792, ENT000027795, ENT000027797, ENT000027800; 11:57, 2 q ENT000027864, ENT000027874; 1ठ ENT000027868; 3우, ENT000027970, ENT000027968, ENT000027966,

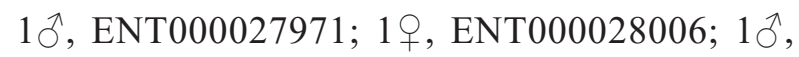
ENT000028022; 11.ii.2016, 1 ENT000028352; 1 을 ENT000028456; 1 오 ENT000028724, $1 \widehat{\jmath}$, ENT000028717; 2ㅇ, ENT000028577, ENT000028575; 21 ㅇ, ENT000028685, ENT000028683, ENT000028682, ENT000028681, ENT000028680, ENT000028676, ENT000028675, ENT000028671, ENT000028668, ENT000028666, ENT000028663, ENT000028661, ENT000028651, ENT000028649, ENT000028647, ENT000028643, ENT000028642, ENT000028640, ENT000028637, ENT000028633, ENT000028625, 1ठ, ENT000028630; 13:55, 6우, ENT000028620, ENT000028616, ENT000028615, ENT000028606, ENT000028589; ENT000028586; 11:30, 9ㅇ, ENT000028513, ENT000028503, ENT000028502, ENT000028493, ENT000028491, ENT000028489, ENT000028488, ENT000028485, ENT000028482; 0752'49”N, 3651'51'”, 19.ii.2016, 1961 m, Plot 2, Dani, 10:50, 1 q ENT000027837; $11: 30,3$ ENT000027763, ENT000027771, ENT000027773; 12:35, 2ㅇ ENT000027908, ENT000027913; Siel, 12:15, 1q, ENT000028080; 11:30, 4,ENT000028071, ENT000028052,
ENT000028051, ENT000028050; 10:50 1ㅇ ENT000028253; Limmu-Kosa, 0757'12”N, 3652'46”'E, 9.ii.2016, 1777 m, coll. L. Geeraert, 10:06, 1ठ̄, ENT000027956.

Stomoxys taeniatus Bigot (Figs 1d-e) ETHIOPIA: Jimma, Limmu-Kosa, 0757’12”N, 3652'46”'E, 9.ii.2016, 1777 m, coll. L. Geeraert, Random sweepnetting, Plantation coffee management system, 1q, ENT000027953; 075' 57'N, 36 51'57'"E, 4.ii.2016, 1786 m, $1{ }^{\lambda}$, ENT000028756; 0755'18”N, 3652'14”'E, 10.ii.2016, $1908 \mathrm{~m}, 1 \widehat{\jmath}$, ENT000028471; 1우, ENT000028317; 05.ii.2016, 2ㅇ, ENT000027784, ENT000027785; Girmo, 0752'51'N, 36 51'47’E, 11.ii.2016, $1920 \mathrm{~m}$, coll. L. Geeraert, 1 , ENT000028618; $07^{\circ} 52^{\prime} 51^{\prime \prime N}, 36^{\circ} 51^{\prime} 47^{\prime \prime} \mathrm{E}$, 24.ii.2016, $1920 \mathrm{~m}$, coll. L. Geeraert, 1ㅇ, ENT000027873.

Stomoxys varipes (Bezzi) - ETHIOPIA: Jimma, Girmo, 0752'51”N, 3651'47”E, 11.ii.2016, 1920 m, coll. L. Geeraert, Random sweepnetting, Semi-forest coffee management system, 1 , ENT000028547.

\section{Conopidae}

Stylogaster nitens Brunneti: ETHIOPIA: Jima, Girmo, 0752'49'’N, 3651'51'’E, 19-24.ii.2016, 1961 m, coll. L. Geeraert, Malaise trap, semi-forest coffee management system; Plot 2, 3 ô; 24-29. ii.2016, $1 \hat{\jmath}$.

Stylogaster westwoodi Smith - ETHIOPIA: Jimma, Limmu-Kosa, 0757'12'N, 652'46”E, 0305.II.2016, 1777 m, coll. L. Geeraert, Malaise trap; Plantation coffee management system; Plot 1, 2ک; 05-09.ii.2016, 1ठ; 11-16.ii.2016, 8ठิ, 1q; 23-23. ii. $2016,3 \hat{\jmath}$.

Stylogaster sp.; ETHIOPIA: Jimma, LimaKosa, 0757'12'N, 3652'46’E, 11-16.iii.2016, $1777 \mathrm{~m}$, coll. L. Geeraert, Malaise trap, Plantation coffee management system; Plot 1, 1 q. 


\section{ACKNOWLEDGMENTS}

We would like to thank Viviane Rodrigues de Sousa (MN/UFRJ) who helped with the photographs using the Auto-Montage Syncroscopy and the dissection of the eggs, of the eggs; Inge Dox who helped with the sorting, pinning, and labeling of the specimens; Siel Wellens (BINCO npo), Daniel Damtew (Jimma University) and Yalew for their help in collecting the specimens; the farmers who allowed us to work in their forests; and Horizon Plantations PLC for permission to access the Limmu Coffee Farm. This research was financially supported by the Flemish Interuniversity Council (VLIR-UOS) and by the Flemish Fund for Scientific Research (FWO) (project G.0565.17).

\section{AUTHOR CONTRIBUTIONS}

MSC identified the material and wrote the manuscript; KJ and LG collected the material and wrote the manuscript; RML and APVA identified the material and prepared the photos and the table; $\mathrm{KJ}$ revised the manuscript; all authors approved the final version of the manuscript.

\section{REFERENCES}

COURI MS AND BARROS GPS. 2010. Diptera hosts of Stylogaster Macquart (Diptera, Conopidae) from Madagascar and South Africa. Rev Bras Entomol 54: 361366.
COURI MS AND PONT AC. 2006. Eggs of Stylogaster Macquart (Diptera: Conopidae) on Madagascan muscids (Diptera: Muscidae). Proc Calif Acad Sci 57: 473-478.

COURI MS, PONT AC AND KIRK-SPRIGGS A. 2013. New Muscidae (Diptera) hosts of Stylogaster Macquart (Diptera: Conopidae) from the Afrotropical Region. Afr Invertebr 54: 401-408.

EMDEN FI. 1951. Muscidae: C-Scatophaginae, Anthomyiinae, Lispinae, Fanniinae and Phaoninae. Ruwenzori Expedition 1934-35(2): 325-710.

SKEVINGTON JH, THOMPSON FC AND CAMRAS S. 2010. Conopidae (Thick-Headed Flies). In: Brown BV, Borkent A, Cumming JM, Wood DM, Woodley NE and Zumbado M (Eds), Manual of Central American Diptera. NRC Research Press, Ottawa, 2, p. 847-855.

SMITH KGV. 1967. The biology and taxonomy of the genus Stylogaster Macquart, 1835 (Diptera: Conopidae, Stylogasterinae) in the Ethiopian and Malagasy regions. Proc R Entomol Soc Lond 199: 47-69.

SMITH KGV. 1980. Family Conopidae. In: Crosskey RW (Eds), Catologue of the Diptera of the Afrotropical Region. British Museum (Natural History), London, p. 511-517.

STUCKENBERG BR. 1963. A study on the biology of the genus Stylogaster, with the description of a new species from Madagascar. Rev Zool Bot Afr 68: 251-275.

STUKE JH. 2012. A revision of Afrotropical Species of Stylogaster Macquart (Diptera: Conopidae), with descriptions of twenty-one new species and an identification key. Afr Invertebr 53: 267-354.

STUKE JH. 2017. Conopidae (Diptera). In: Brill EJ (Ed), World Catalogue of Insects. Brill, Boston, 15, 380 p.

ZIELKE E. 1971. Revision der Muscinae der aethiopischen Region. Ser Entomol 7: 1-199.

ZUMPT F. 1973. The Stomoxyine Biting Flies of the World. Diptera: Muscidae. Taxonomy, Biology, Economic Importance and Control Measures. Gustav Fisher, Sttutgart, Germany. viii +175 p. 